\title{
IAMJ
}

INTERNATIONAL

AYURVEDIC

MEDICAL JOURNAL

Review Article

ISSN: 2320-5091

Impact Factor: 6.719

\section{AGATHIYAR KUZHAMBU (HERBO-MINERAL SIDDHA FORMULATION)}

\author{
Rakulini Raveendran ${ }^{1}$, Sounthararajan Kalaiselvi ${ }^{2}$, Sundararajan $\mathbf{S}^{3}$ \\ ${ }^{1}$ PG Scholar, MD (S), Government Siddha Medical College, Palayamkottai affiliated with \\ Dr. M.G.R Medical University, \\ ${ }^{2}$ Senior Lecturer, Unit of Siddha Medicine, University of Jaffna, \\ ${ }^{3}$ Reader \& HOD, Dept. of Noi Naadal, Government Siddha Medical College, Palayamkottai affiliated with \\ Dr. M.G.R Medical University,
}

Corresponding Author: r.rakulini@gmail.com

https://doi.org/10.46607/iamj3009042021

(Published Online: April 2021)

Open Access

(C) International Ayurvedic Medical Journal, India 2021

Article Received: 31/03/2021 - Peer Reviewed: 02/04/2021 - Accepted for Publication: 07/04/2021

Check for updates

\begin{abstract}
Introduction: Agathiyar kuzhambu (A.k) is a herbo-mineral siddha formulation. It is explained in the Siddha Vaithiya Thiraddu Book. In Siddha Medicine, there are 32 internal \& external medicines are explained in the Siddha Literatures. Agathiyar kuzhambu is a one form of the internal medicine which is commonly used in purgation therapy. It contains 11 ingredients. Agathiyar kuzhambu has different herbo- mineral ingredients which are having wide range of therapeutic uses. It is commonly used in Siddha OPDs. Aim: The researcher is going to develop the documentary evidence for the effectiveness of Agathiyar kuzhambu. Results and Discussion: Six ingredients herbs have hot potency and Pungent efficacy. These have many pharmacological actions. Commonly, these ingredients are possessed antioxidant, anti-microbial, hepatoprotective, anti-inflammatory, anti-fungal, anthelmintic, anti-diabetic and anti -hypertensive activity. In Siddha, these ingredients and 5 minerals and metals ingredients were used in many disease conditions. Lots of adjuvants of Agathiyar kuzhambu have hot potency and pungent efficacy. Some of adjuvants have cool potency and sweet efficacy. In Siddha, these adjuvants were used in many disease conditions. Conclusion: According to the above study, Agathiyar kuzhambu may be act as stimulant and osmotic purgative at the same time it can use as a treatment aspect of many diseases. This study gives documentary evidence of Agathiyar kuzhambu as a purgative \& possess many pharmacological activities in Siddha medicine.
\end{abstract}


Keywords: Agathiyar kuzhambu, Siddha Medicine, Purgative, Croton tiglium

\section{INTRODUCTION}

Agathiyar kuzhambu (A.k) is a herbo-mineral siddha formulation. It is explained in the Siddha Vaithiya Thiraddu Book. In Siddha Medicine, there are 32 internal \& external medicines are explained in the Siddha Literatures. Agathiyar kuzhambu is a one form of the internal medicine which is commonly used in purgation therapy. It contains 11 ingredients; 6 herbal ingredients: Ferula asafoetida H.Karst, Brassica nigra (L.) K.Koch, Aconitum ferox Wall. ex Ser., Trachyspermum ammi (L.) Sprague, Nigella sativa L. and Croton tiglium L. and 5 minerals and metals: Rasam (Mercury quicksilver), Inthuppu (Sodium chloride impura), Venkaram (Sodium biborate), Manosilai (Arsenic disulphidum bisulpuret of arsenic realgar (Red orpiment)) and Aritharam (Trisulphate of arsenic (Yellow orpiment)). It is used to treat a wide variety of diseases. [1]

4448 diseases are mentioned in Agasthiyar 2000, Angathipatham, Noi Naadal Noi Muthal Naadal part I and Theriyar vaakadam. Diseases are classified according to anatomical structures of body parts, tri humors, clinical sign and symptoms and physical constituents. ${ }^{[2]}$ According to the Siddha Medicine, methods of treatment are explained in three different kinds. They are Divine treatment, Rational treatment and Surgical treatment. Treatments are classified according to the therapy. There are Purgative therapy, Emetic therapy, Fasting therapy, Steam therapy, Oleation therapy, Physical therapy (Thokkanam), Solar therapy, Bloodletting therapy, Diseases curable with herbal medicines and Diseases curable with metals and mineral preparation. Purgation therapy is a base line treatment. It is given during the month of Aani and aadi (mid-June to mid-August). The decoction, pastes, kuzhambu and medicated oil are preferred for purgation. ${ }^{[3]}$

Elimination of vitiated Doshas through rectum is called purgation therapy. Proper administrated purificatory drug will give clarify of the mind, strengthens sense organs, gives stability to tissues, improve digestive power, reduces aging etc...

Ingredients of Agathiyar Kuzhambu ${ }^{\text {[1] }}$

Ferula asafoetida H.Karst $\quad 5 \mathrm{~g}$

Brassica nigra (L.) K.Koch $\quad 5 \mathrm{~g}$

Purified Aconitum ferox Wall. ex Ser. $5 \mathrm{~g}$

Trachyspermum ammi (L.) Sprague $\quad 5 \mathrm{~g}$

Nigella sativa $\quad$ L. $\quad 5 \mathrm{~g}$

Purified Rasam (Mercury quicksilver) $5 \mathrm{~g}$

Purified Inthuppu (Sodium chloride impura) $5 \mathrm{~g}$

Purified Venkaram (Sodium biborate) $5 \mathrm{~g}$

Purified Manosilai (Arsenic disulphidum bisulpuret of arsenic realgar (Red orpiment)) $\quad 5 \mathrm{~g}$

Purified Aritharam (Trisulphate of arsenic (Yellow orpiment)) $5 \mathrm{~g}$

Purified Croton tiglium L. $\quad 50 \mathrm{~g}$

\section{Preparation of Agathiyar Kuzhambu ${ }^{[1]}$}

All above crude and raw drugs were purified and finely powdered separately except Croton tiglium L. The quantity of each ingredients was accurately weighted and mixed and grained all together in grindstone. Weighted Croton tiglium L. was divided into two parts. One part was added directly, and another part was fried with castor oil, then both were grinded in grind stone. Finally, all ingredients were grained all together and preserved in the bottle.

\section{Dosage form, vehicle and disease conditions ${ }^{[1]}$}

\begin{tabular}{|l|l|l|}
\hline Dosage form & Vehicle & Disease conditions \\
\hline Internal $-130 \mathrm{mg}$ & Chukku (Zingiber officinale Roscoe) katkam & Fever \\
\hline & Kadukkai (Terminalia chebula Retz.) kudineer & Cough \\
\hline $\begin{array}{l}\text { Chukku (Zingiber officinale Roscoe) \& Milaku (Piper } \\
\text { nigrum } \text { L.) powder }\end{array}$ & Chills with fever \\
\hline & $\begin{array}{l}\text { Ghee, Buffalo milk } \\
\text { Milaku (Piper nigrum L.) powder with white onion } \\
\text { juice }\end{array}$ & $\begin{array}{l}\text { Bleeding piles } \\
\text { Anaemia, Veppup paavai, Neerampal, } \\
\text { Sokai }\end{array}$ \\
\hline
\end{tabular}




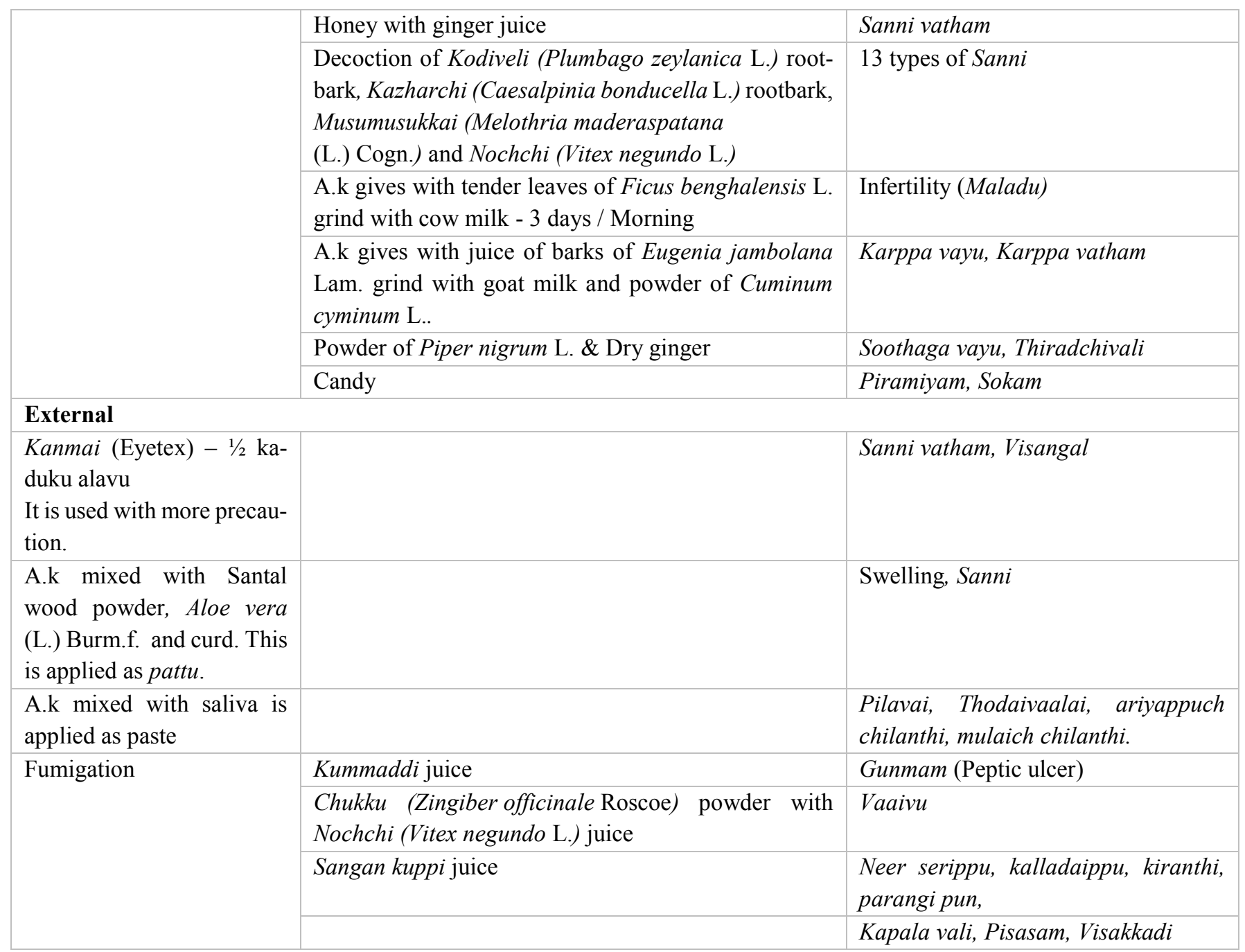

Pathiyam: Ghee, Murukkam pinchu, Vaalai pinchu, Naaraththai and Thuthuvalai. Morum sorum. ${ }^{[1]}$

Excessive Purgation: Use decoction of Cuminum cyminum L., and tamarind or lemon / burned Acorus calamus L. / Butter milk with rice.

\section{Butter milk}

One cup $(245 \mathrm{ml})$ of cultured buttermilk provides the following nutrients: Calories: 98, Protein: 8 grams, Carbs: 12 grams, Fat: 3 grams, Fiber: 0 grams, Calcium: $22 \%$ of the Daily Value (DV), Sodium: $16 \%$ of the DV, Riboflavin: $29 \%$ of the DV, Vitamin B12:22\% of the DV and Pantothenic acid: $13 \%$ of the DV.

Agathiyar kuzhambu has different herbo- mineral ingredients which are having wide range of therapeutic

\section{Results}

uses. It is commonly used in Siddha OPDs. So, the researcher is going to develop the documentary evidence for the A.k.

\section{Material and Methods}

Research Type: Literature Review

Research Design: Collection of data from the available authenticated Tamil Siddha texts and journal articles. All data (Part use, taste, potency, efficacy, phytochemical, pharmacological action, chemical constituents and medicinal uses) were collected of all ingredients of A.k and adjuvants of A.k.

Statistical analysis: Collected data were processed and statistically analysed by simple statistical method using MS. Excel 2019. 
Table 1: Description of raw materials of Agathiyar Kuzhambu ${ }^{[4][5]}$

\begin{tabular}{|c|c|c|c|c|c|c|c|c|}
\hline \multirow{2}{*}{$\begin{array}{l}\text { Botanical } \\
\text { Name }\end{array}$} & \multirow{2}{*}{$\begin{array}{l}\text { Tamil } \\
\text { Name , } \\
\text { English } \\
\text { Name }\end{array}$} & \multirow{2}{*}{$\begin{array}{l}\text { Family } \\
\text { Name }\end{array}$} & \multirow{2}{*}{$\begin{array}{l}\text { Parts } \\
\text { used }\end{array}$} & \multicolumn{3}{|c|}{ Siddha properties } & \multirow{2}{*}{$\begin{array}{l}\text { Pharmacological } \\
\text { action }\end{array}$} & \multirow[t]{2}{*}{ Medicinal uses } \\
\hline & & & & Taste & $\begin{array}{l}\text { Po- } \\
\text { tency }\end{array}$ & $\begin{array}{l}\text { Effi- } \\
\text { cacy }\end{array}$ & & \\
\hline $\begin{array}{l}\text { Fer- } \\
\text { ula asa- } \\
\text { foet- } \\
\text { ida } \text { H.Kar } \\
\text { st }\end{array}$ & $\begin{array}{l}\text { Perumka- } \\
\text { yam / } \\
\text { Asafae- } \\
\text { tida }\end{array}$ & $\begin{array}{l}\text { Umbel- } \\
\text { liferae }\end{array}$ & $\begin{array}{l}\text { Gum } \\
\text { resin }\end{array}$ & Bitter & Hot & $\begin{array}{l}\text { Pun- } \\
\text { gent }\end{array}$ & $\begin{array}{l}\text { Stimulant, Car- } \\
\text { minative, Anti- } \\
\text { spasmodic, Ex- } \\
\text { pectorant, Laxa- } \\
\text { tive, Anthelmen- } \\
\text { tic, Diuretic, } \\
\text { Aphrodisiac, } \\
\text { Emmenagogue }\end{array}$ & $\begin{array}{l}\text { It is used for Dental diseases, } \\
\text { Thantha moolarogam, All } \\
\text { snake poison, Kirumi, Indi- } \\
\text { gestion, Vathathikkam, } \\
\text { Uthavarththavaatham, Yoni } \\
\text { rogam, Iruthaya vayu, } 8 \text { types } \\
\text { of Gunmam, Uthara rogam, } \\
\text { Karppa vippuruthi, Soolai, } \\
\text { Iraththa kirumi, Kapha dis- } \\
\text { eases and Body pain[7]. }\end{array}$ \\
\hline $\begin{array}{l}\text { Bras- } \\
\text { sica nigra } \\
\text { (L.) } \\
\text { K.Koch }\end{array}$ & $\begin{array}{l}\text { Kadugu / } \\
\text { Black } \\
\text { Mustard } \\
\text { Seed }\end{array}$ & $\begin{array}{l}\text { Brassi- } \\
\text { caceae }\end{array}$ & Seeds & $\begin{array}{l}\text { Pun- } \\
\text { gent }\end{array}$ & Hot & $\begin{array}{l}\text { Pun- } \\
\text { gent }\end{array}$ & $\begin{array}{l}\text { Emetic, Stimu- } \\
\text { lant, Rubefa- } \\
\text { cient, Vesicant, } \\
\text { Digestive, Diu- } \\
\text { retic }\end{array}$ & $\begin{array}{l}\text { It is used to treat Sinusitis, } \\
\text { Headache due to cough, } \\
\text { Phlegm, Indigestion, ab- } \\
\text { dominal pain, Vatha thodam, } \\
\text { Kaanak kadi, Vatha kapham, } \\
\text { Kirakani, Tri-humors, Seetha } \\
\text { kaduppu, Joint diseases, gid- } \\
\text { diness and hiccough. }\end{array}$ \\
\hline $\begin{array}{l}\text { Aconi- } \\
\text { tum ferox } \\
\text { Wall. ex } \\
\text { Ser. }\end{array}$ & $\begin{array}{l}\text { Naabi / } \\
\text { Indian ac- } \\
\text { onite }\end{array}$ & $\begin{array}{l}\text { Ranun- } \\
\text { cula- } \\
\text { ceae }\end{array}$ & $\begin{array}{l}\text { Dry } \\
\text { rhi- } \\
\text { zome }\end{array}$ & Bitter & Hot & $\begin{array}{l}\text { Pun- } \\
\text { gent }\end{array}$ & $\begin{array}{l}\text { Diaphoretic, Di- } \\
\text { uretic, Anti-peri- } \\
\text { odic, Anodyne, } \\
\text { Anti-diabetic, } \\
\text { Anti-phlogistic, } \\
\text { Antipyretic, Nar- } \\
\text { cotic, Sedative }\end{array}$ & $\begin{array}{l}\text { It is used for Vatha Kaduppu, } \\
\text { Indigestion, Kapha diseases, } \\
\text { Kuddam, Peptic ulcer and } \\
\text { Scorpion poison. }\end{array}$ \\
\hline $\begin{array}{l}\text { Tra- } \\
\text { chysper- } \\
\text { mum am } \\
\text { mi (L.) } \\
\text { Sprague }\end{array}$ & $\begin{array}{l}\text { Omam / } \\
\text { The Bish- } \\
\text { ops weed }\end{array}$ & $\begin{array}{l}\text { Apiacea } \\
\mathrm{e}\end{array}$ & & $\begin{array}{l}\text { Pun- } \\
\text { gent }\end{array}$ & Hot & $\begin{array}{l}\text { Pun- } \\
\text { gent }\end{array}$ & $\begin{array}{l}\text { Stomachic, Anti- } \\
\text { spasmodic, Car- } \\
\text { minative, Anti- } \\
\text { septic, Stimulant, } \\
\text { Astringent, } \\
\text { Tonic, Sialo- } \\
\text { gogue }\end{array}$ & $\begin{array}{l}\text { It is used for Seetha suram, } \\
\text { Indigestion, Abdominal dis- } \\
\text { comfort, Purgative, Seetha } \\
\text { Pathi, Suvasakasam, Dental } \\
\text { diseases and Rectal pain }\end{array}$ \\
\hline $\begin{array}{l}\text { Ni- } \\
\text { gella sa- } \\
\text { tiva } \mathrm{L} \text {. }\end{array}$ & $\begin{array}{l}\text { Karum- } \\
\text { cheeraka } \\
m / \text { Black } \\
\text { cumin, } \\
\text { Small } \\
\text { fennel }\end{array}$ & $\begin{array}{l}\text { Ranun- } \\
\text { cula- } \\
\text { ceae }\end{array}$ & Seeds & Bitter & Hot & $\begin{array}{l}\text { Pun- } \\
\text { gent }\end{array}$ & $\begin{array}{l}\text { Carminative, Di- } \\
\text { uretic, Emmena- } \\
\text { gogue, Galacta- } \\
\text { gogue, Anthel- } \\
\text { mintic, Sto- } \\
\text { machic, Parasiti- } \\
\text { cide, Emollient }\end{array}$ & $\begin{array}{l}\text { It is used for Mandai karap- } \\
\text { pan, Wounds, Sinusitis, Body } \\
\text { heat, Head pain, Eye diseases, } \\
\text { Sirangu, Abdominal discom- } \\
\text { fort, Peptic ulcer, Chest pain, } \\
\text { Cough, Vomiting, Nausea, } \\
\text { Swelling and jaundice. }\end{array}$ \\
\hline $\begin{array}{l}\text { Cro- } \\
\text { ton tig- } \\
\text { lium } \mathrm{L} .\end{array}$ & $\begin{array}{l}\text { Ner- } \\
\text { valam/ } \\
\text { Purgative } \\
\text { croton }\end{array}$ & $\begin{array}{l}\text { Euphor- } \\
\text { biaceae }\end{array}$ & Seeds & Bitter & Hot & $\begin{array}{l}\text { Pun- } \\
\text { gent }\end{array}$ & $\begin{array}{l}\text { Purgative, Stim- } \\
\text { ulant, Rubefa- } \\
\text { cient }\end{array}$ & $\begin{array}{l}\text { It is used for chronic constipa- } \\
\text { tion, Rectal and bdominal dis- } \\
\text { eases, Vatha diseases, Visama } \\
\text { sanni paatham and Body pain }\end{array}$ \\
\hline
\end{tabular}


Above six ingredients have hot potency and Pungent efficacy. In Siddha, these ingredients were used in many disease conditions.

Table 2: Description of Minerals of Agathiyar Kuzhambu ${ }^{[6]}$

\begin{tabular}{|c|c|c|c|}
\hline $\begin{array}{l}\text { Scientific Name of } \\
\text { Minerals }\end{array}$ & $\begin{array}{l}\text { Tamil Name / } \\
\text { English Name }\end{array}$ & Pharmacological action & Medicinal uses \\
\hline Mercury quicksilver & $\begin{array}{l}\text { Rasam / Mer- } \\
\text { cury/ Hydrargy- } \\
\text { rum }\end{array}$ & $\begin{array}{l}\text { Tonic, Alternative, Nutrient, } \\
\text { Laxative, anti-bilious, Siala- } \\
\text { gogue, Diuretic, Deobstruent, } \\
\text { Antiseptic }\end{array}$ & $\begin{array}{l}\text { Purify the blood, increase blood and semen, } \\
\text { Appetizer, Antiseptic, improve concentra- } \\
\text { tion, Nervine tonic, relax the mind, Delay } \\
\text { aging }\end{array}$ \\
\hline $\begin{array}{l}\text { Sodium chloride im- } \\
\text { pure } \\
\text { Or } \\
\text { Sodii chloridum im- } \\
\text { pura }\end{array}$ & $\begin{array}{l}\text { Inthuppu / Rock } \\
\text { Salt }\end{array}$ & $\begin{array}{l}\text { Laxative, Purgative, Carmina- } \\
\text { tive, Diuretic, Appetizer }\end{array}$ & $\begin{array}{l}\text { It is used for } 8 \text { types of Gunmam, Alasam, } \\
\text { Asirkkaram, Kapha piththam, Kaphaathik- } \\
\text { kam, Narambukiranthi, Constipation, Poi- } \\
\text { son and kapha upsthamsam. It gives relief } \\
\text { from thirst, Nethira kaasam, Bleeding piles } \\
\text { and Toxic bites. }\end{array}$ \\
\hline $\begin{array}{l}\text { Sodium biborate, So- } \\
\text { dium Bi Boras }\end{array}$ & $\begin{array}{l}\text { Venkaram / } \\
\text { Sodii boras, Bo- } \\
\text { rax }\end{array}$ & $\begin{array}{l}\text { Refrigerant, Antiseptic, Lithon- } \\
\text { triptic, Diuretic, Emmenagogue, } \\
\text { Astringent, Sedative }\end{array}$ & $\begin{array}{l}\text { It is used to treat psoriasis, } 8 \text { types of Gun- } \\
\text { mam, Bleeding piles, Urinary tract infec- } \\
\text { tion, Kaphathikkam, Dental diseases, Toxic } \\
\text { bites and Sanni paatham. }\end{array}$ \\
\hline $\begin{array}{l}\text { Arsenic disulphidum } \\
\text { bisulpuret of arsenic } \\
\text { realgar } \\
\& \\
\text { Trisulphate of arsenic }\end{array}$ & $\begin{array}{l}\text { Manosilai / Red } \\
\text { orpiment } \\
\& \\
\text { Aritharam / Yel- } \\
\text { low orpiment }\end{array}$ & Alterative, Tonic, Febrifuge & $\begin{array}{l}\text { It gives relief from Saruma kuttam, Chills } \\
\text { with fever, Bronchial asthma, Silanthi } \\
\text { vidam, Kaasam, Kapha diseases, Eye dis- } \\
\text { eases and Urinary tract infections. }\end{array}$ \\
\hline
\end{tabular}

In Siddha, these ingredients were used in many disease conditions.

Table 3: Pharmacological action of raw material of Agathiyar Kuzhambu

\begin{tabular}{|c|c|c|c|c|c|c|}
\hline Pharmacological activity & $\begin{array}{l}\text { Ferula asa- } \\
\text { foetida } \\
\text { H.Karst }^{[7]}\end{array}$ & $\begin{array}{l}\text { Brassica } \\
\text { nigra (L.) } \\
\text { K.Koch }^{[8]}\end{array}$ & $\begin{array}{l}\text { Aconitum } \\
\text { ferox Wall. ex } \\
\text { Ser. }{ }^{[9][10]}\end{array}$ & $\begin{array}{l}\text { Trachyspermum } \\
\begin{array}{lll}\text { ammi } & \text { (L.) Sprague } \\
{[11][12]} & \end{array}\end{array}$ & $\begin{array}{l}\text { Nigella } \\
\text { sativa L. } \\
{[13]}\end{array}$ & $\begin{array}{l}\text { Croton tig- } \\
\text { lium L } \\
{[14][15]}\end{array}$ \\
\hline Antioxidant & $*$ & $*$ & - & $*$ & $*$ & $*$ \\
\hline Antimicrobial & $*$ & - & - & $*$ & $*$ & $*$ \\
\hline Antiviral & $*$ & - & - & - & - & - \\
\hline Antifungal & $*$ & - & - & $*$ & $*$ & - \\
\hline Anticarcinogenic & $*$ & - & - & - & $*$ & - \\
\hline Anticancer & $*$ & - & - & - & $*$ & \\
\hline Anticytotoxicity & $*$ & - & - & $*$ & - & - \\
\hline Anthelmintic & $*$ & $*$ & - & $*$ & - & - \\
\hline Antagonistic effect & $*$ & - & - & - & - & - \\
\hline Antidiabetic & $*$ & $*$ & - & - & $*$ & \\
\hline Antispasmodic & $*$ & - & - & $*$ & - & - \\
\hline Antihypertensive & $*$ & - & - & $*$ & $*$ & - \\
\hline Anti-lithiasis & - & - & - & $*$ & - & - \\
\hline Anti-filarial & - & - & - & $*$ & - & - \\
\hline Anti- pyretic & - & - & $*$ & - & - & - \\
\hline Anti-inflammatory & - & $*$ & - & - & $*$ & $*$ \\
\hline
\end{tabular}




\begin{tabular}{|c|c|c|c|c|c|c|}
\hline Anti-tumor activity & - & - & - & - & - & * \\
\hline Anti-HIV activity & - & - & - & - & - & $*$ \\
\hline Analgesic & - & - & $*$ & - & $*$ & $*$ \\
\hline Antinociceptive effect & - & - & - & - & - & $*$ \\
\hline Hypolipidemic & - & - & - & $*$ & - & - \\
\hline Antiparasitic Effects & - & - & - & $*$ & - & - \\
\hline Molluscicidal & $*$ & - & - & - & - & $*$ \\
\hline Nematicidal & - & - & - & $*$ & - & - \\
\hline Relaxant & $*$ & - & - & - & - & - \\
\hline Neuroprotective & $*$ & - & - & - & $*$ & - \\
\hline Broncho-dilating actions & - & - & - & $*$ & - & - \\
\hline Diuretic & - & - & - & $*$ & - & - \\
\hline $\begin{array}{l}\text { Immunomodulatory ac- } \\
\text { tivity }\end{array}$ & - & - & - & - & $*$ & - \\
\hline Cardiovascular activity & - & $*$ & - & $*$ & $*$ & - \\
\hline Gastro-protective activity & - & & - & $*$ & $*$ & - \\
\hline $\begin{array}{l}\text { Hepato-protective activ- } \\
\text { ity }\end{array}$ & $*$ & $*$ & - & $*$ & $*$ & - \\
\hline Nephroprotective activity & - & $*$ & - & - & $*$ & - \\
\hline Pulmonary protective & - & $*$ & - & - & $*$ & - \\
\hline Anti-asthmatic activity & - & - & - & - & $*$ & - \\
\hline
\end{tabular}

Above six ingredients have many pharmacological actions. Commonly, these ingredients are possessed antioxidant, anti-microbial, hepatoprotective, anti-inflammatory, anti-fungal, anthelmintic, anti-diabetic and anti -hypertensive activity.

\section{DISCUSSION}

Purgatives either provide semisolid stool or lead to watery evacuation. Purgative drugs are classified into 4: Bulk forming, Stool softener, Stimulant purgative and Osmotic purgative. Stimulant purgatives are stimulating peristalsis by irritant action on intestinal mucosa and also stimulate colonic electrolytes and fluid secretion by altering absorptive and secretory activity of mucosal cells. Osmotic purgatives are soluble inorganic salts, that increases the faecal bulk by retaining water osmotically and distend the bowel increasing peristalsis indirectly. It is an act on small as well as large intestine. A.k contains 11 ingredients; 6 herbal ingredients: Ferula asafoetida, Brassica nigra, Aconitum ferox, Trachyspermum ammi, Nigella sativa and Croton tiglium. All above six ingredients have hot potency and Pungent efficacy. These have many pharmacological actions.
Commonly, these ingredients are possessed antioxidant, anti-microbial, hepatoprotective, anti-inflammatory, anti-fungal, anthelmintic, anti-diabetic and anti hypertensive activity. In Siddha, these ingredients were used in many disease conditions.

One of the studies said that crotonoleic acid in the ethanol extract of croton seeds possessed significant purgative effect in experimental animals. Crotonoleic acid which is a mixture of croton resin with inactive fatty acids may act as a powerful irritant to the intestinal mucosa and thereby causing enteropooling effect. In vitro studies using the extract showed the presence of muscarnic activity. Hence it can be presumed that the stimulation of the gut cause by the extract may partly be due to the activation of muscarnic receptors present. However, the present findings may be of support to the use of Croton tiglium as a purgative in traditional medicine. ${ }^{[20]}$

5 minerals and metals are used. In Siddha, these ingredients were used in many disease conditions. Lots of adjuvants of A.k have hot potency and pungent efficacy. Some of adjuvants have cool potency and sweet 
efficacy. In Siddha, these adjuvants were used in many disease conditions.

Rock salt: It may be act as an osmotic purgative.

Stimulant purgatives can lead to purgation resulting in fluid and electrolyte imbalance and hypokalemia. It is neutralized by buttermilk with rice.

\section{CONCLUSION}

According to the above study, Agathiyar kuzhambu may be act as stimulant and osmotic purgative at the same time it can be used as a treatment aspect of many diseases. This study gives documentary evidence of Agathiyar kuzhambu as a purgative \& possess many pharmacological activities in Siddha medicine.

\section{REFERENCES}

1. Kuppusaamy, N. K. Uththamarajan, K. S., Siddha Vaithiya Thiraddu. Indian medicine - Homeopathy, 2009. $3^{\text {rd }}$ ed. pp. 168-173.

2. Rakulini, R. "Classification of 4448 Diseases in Siddha System of Medicine - A Review" Journal of Complementary and Alternative Medical Research. 2020; 9(2): 46-54.

3. Uthamaroyan, CS. A compendium of Siddha doctrine. Dept. of Indian Medicine \& Homeopathy, Chennai. 2005. $1^{\text {st }} \mathrm{ed}$.

4. Murugesha muthaliyar, K. S., Gunapadam (Muligai vakuppu), Materia Medica Part I. Government press. 1936. $1^{\text {st }}$ ed.

5. Vivian, S., 2008. Gunapadam II. Harinee enterprises. $1^{\text {st }}$ ed.

6. Thiyagarajah, R. Gunapadam Thathu jeeva vaguppu Part 2 -3, Dept. of Indian Medicine \& Homeopathy, Chennai. 2004. $4^{\text {th }}$ ed.

7. Poonam, M. Sharadha, B. Ferula asafoetida: Traditional uses and pharmacological activity, Pharmacogn Rev. 2012 Jul-Dec;6(12): 141-146.

8. Ali Esmail Al-Snafi, The pharmacological importance of Brassica nigra and Brassica rapa grown in Iraq. Journal of Pharmaceutical Biology. 5(4). 2015. 240-253.

9. Mukesh Kr. Singh et al., (2012) Int.J. Res. Pharm. Sci, 3(2), 242-246.

10. Liu Z et al./ IJPR (2012), 11 (2): 611-620

11. Singh, G., S. Maurya, C. Catalan and M.P. de Lampasona, 2004. Chemical constituents, antifungal and antioxidative effects of ajwain essential oil and its acetone extract. J. Agric. Food Chem., 52: 3292-3296.
12. Sonal Dubey, Pankaj Kashyap, Trachyspermum ammi: A Review on its Multidimensional Uses in Indian Folklore Medicines, Res.J.Med. Plant, 9 (8): 368-374, 2015.

13. Krishnapura, S. Cumin (Cuminum cyminum) and black cumin (Nigella sativa) seeds: traditional uses, chemical constituents and nutraceutical effects, Food Quality and Safety, 2018, 2, 1-16.

14. T.Dey et al. (2015) / Int.J.Curr. Res. Biosci. Plant Biol. 2015, 2(7): 124-128.

15. Simran, S. Indhumathi, P. Meenakshi, S. M. An overview of the biological and chemical perspectives of $\mathrm{CrO}$ ton tiglium, Der Pharmacia Lettre, 2016, 8(19): 324-328.

16. Zoheir A Damanhouri, Aftab Ahmad, A review on therapeutic potential of Piper nigrum L. (Black Pepper): The King of Spices, Medicinal \& Aromatic Plants 2014, 3(3), 3-6.

17. Vishnukanta, Rana, A. C. Plumbago zeylanica: A phytopharmacological review, IJPSR, 2011; Vol.2 (2): 247255.

18. Gopukumar, S.T. Prasetha, P. K. Ficus benghalensis linn- The Sacred Indian medicinal tree with potent pharmacological remedies, Int. J. Pharm. Sci. Rev. Res., 32(1), 2015; 223-227.

19. Abdul khalique, Abdur rauf, An overview of Jamun (Eugenia Jambolana Linn): A Traditional Multipotential Drug, Ind.J.Unani Med., IX, Iss. 2, pp 71-75,2016.

20. Pillai, N. R. Gastrointestinal effects of Croton tiglium in experimental animals, Ancient Science of Life, Vol.18(3\&4), 1999, 205-209.

\section{Source of Support: Nil Conflict of Interest: None Declared}

How to cite this URL: Rakulini Raveendran et al: Agathiyar Kuzhambu (Herbo-Mineral Siddha Formulation). International Ayurvedic Medical Journal \{online\} 2021 \{cited April, 2021\} Available from: http://www.iamj.in/posts/images/upload/869_875.pdf 